\title{
Gene Expression Changes in Venous Segment of Overflow Arteriovenous Fistula
}

\author{
Yasuhiro Hashimoto, ${ }^{1,2}$ Akiko Okamoto, ${ }^{1}$ Hisao Saitoh, ${ }^{1}$ Shingo Hatakeyama, ${ }^{2}$ \\ Takahiro Yoneyama, ${ }^{2}$ Takuya Koie, ${ }^{2}$ and Chikara Ohyama ${ }^{2}$ \\ ${ }^{1}$ Oyokyo Kidney Research Institute, Hirosaki Hospital, Hirosaki 036-8243, Japan \\ ${ }^{2}$ Department of Urology, Hirosaki University Graduate School of Medicine, 5 Zaifucho, Hirosaki, Aomori 036-8562, Japan
}

Correspondence should be addressed to Yasuhiro Hashimoto; bikkuri@opal.plala.or.jp

Received 11 February 2013; Revised 6 April 2013; Accepted 6 April 2013

Academic Editor: Alejandro Martín-Malo

\begin{abstract}
Copyright (C) 2013 Yasuhiro Hashimoto et al. This is an open access article distributed under the Creative Commons Attribution License, which permits unrestricted use, distribution, and reproduction in any medium, provided the original work is properly cited.
\end{abstract}

\begin{abstract}
Aim. The objective of this study was to characterize coordinated molecular changes in the structure and composition of the walls of venous segments of arteriovenous (AV) fistulas evoked by overflow. Methods. Venous tissue samples were collected from 6 hemodialysis patients with AV fistulas exposed to overflow and from the normal cephalic veins of 4 other hemodialysis patients. Total RNA was extracted from the venous tissue samples, and gene expression between the 2 groups was compared using Whole Human Genome DNA microarray $44 \mathrm{~K}$. Microarray data were analyzed by GeneSpring GX software and Ingenuity Pathway Analysis. Results. The cDNA microarray analysis identified 397 upregulated genes and 456 downregulated genes. Gene ontology analysis with GeneSpring GX software revealed that biological developmental processes and glycosaminoglycan binding were the most upregulated. In addition, most upregulation occurred extracellularly. In the pathway analysis, the TGF beta signaling pathway, cytokines and inflammatory response pathway, hypertrophy model, and the myometrial relaxation and contraction pathway were significantly upregulated compared with the control cephalic vein. Conclusion. Combining microarray results and pathway information available via the Internet provided biological insight into the structure and composition of the venous wall of overflow AV fistulas.
\end{abstract}

\section{Introduction}

Arteriovenous (AV) fistulas are very useful for determining optimal blood flow for dialysis, but AV fistulas exposed to overflow are thought to increase cardiac output and cause high-output cardiac failure $[1,2]$.

Measurement of blood flow via an internal shunt was first developed by Krivitski et al., and the monitoring of blood flow via a shunt has since become widespread [3]. We use this technique to monitor the blood flow of AV fistulas at our hospital and correct overflow AV fistulas with surgery.

It is thought that the outflow vein of overflow AV fistulas bears a heavy load: as the vein is exposed to increased arterial flow, the wall dilates, triggering a vascular remodeling process. However, the molecular mechanisms by which the outflow vein is remodeled into a mature fistula remain unclear. By investigating venous remodeling in overflow AV fistulas, candidate genes important to the remodeling process can be discovered and their functional significance investigated. Thus, the identification of relevant genes involved in this process should provide insight into AV fistula maturation.

In this study, we performed a cDNA microarray analysis and compared segments of the venous walls of overflow AV fistulas from 6 hemodialysis patients with the normal cephalic veins of 4 other hemodialysis patients to determine whether there was any difference in their gene expression patterns.

\section{Study Population}

From June 2009 to September 2010, 548 patients underwent hemodialysis at the Oyokyo Kidney Research Institute in Hirosaki, Japan. During that period, 10 patients underwent surgical ligation to correct an overflow AV fistula. When the operation was performed, we retained a sample of the wall of 
the overflow AV fistula (Figure 1). The AV fistula specimens were resected from the wall of the vein close to the AV fistula anastomosis. The study was approved by the Bioethics Committee of Oyokyo Kidney Research Institute, and all patients provided their informed consent to the procedure prior to it being performed.

\section{Inclusion Criteria}

The inclusion criteria were as follows: (1) blood access flow greater than $2.0 \mathrm{~L} / \mathrm{min}$ measured by the color Doppler ultrasound (2) an AV fistula in the lower arm with a distal radiocephalic anastomosis. In total, 6 patients had overflow AV fistulas that met these criteria. The backgrounds of these patients are detailed in Table 1 . We also obtained tissue samples from the lower arm distal cephalic veins of 4 new hemodialysis patients and used these as a control.

\section{Methods}

As noted above, venous tissues were resected from a venous segment of an overflow AV fistula from 6 patients and from a normal cephalic vein from 4 other patients. The surgical specimens were immediately placed in test tubes containing RNAlater (see below for details).

Total RNA was extracted from the venous tissue samples, and gene expression between the 2 groups was compared using Whole Human Genome DNA microarray 44 K (Agilent Technologies, Santa Clara, California). The microarray data were analyzed with GeneSpring GX software and Ingenuity Pathway Analysis.

\section{RNA Isolation}

Surgical specimens were $0.5 \mathrm{~cm}$ or smaller in size and were initially stored in RNA later (Ambion, Austin, TX) overnight at $4 \pm 3^{\circ} \mathrm{C}$ then at $-80^{\circ} \mathrm{C}$ until RNA extraction. Total RNA was extracted using TRIzol reagent (Invitrogen, Carlsbad, CA) according to the manufacturer's instructions. The total RNA was further purified using the Qiagen RNeasy Mini Kit (Qiagen, Valencia, CA) and then extracted. The quantity and quality of the RNA were determined using a Nanodrop ND-1000 spectrophotometer (Thermo Fisher Scientific Inc., Waltham, MA) and an Agilent Bioanalyzer (Agilent Technologies, Palo Alto, CA).

\section{6. cRNA Amplification and Labeling}

Total RNA was amplified and labeled with Cyanine 3 (Cy3) as instructed by the manufacturer of the Agilent Low Input Quick Amp Labeling Kit, one-color (Agilent Technologies, Palo Alto, CA). Briefly, 100 ng of total RNA was reverse transcribed to double-strand cDNA using a poly dT-T7 promoter primer. The primer, template RNA, and quality-control transcripts of known concentration and quality were then denatured at $65^{\circ} \mathrm{C}$ for $10 \mathrm{~min}$ and incubated for 2 hours at $40^{\circ} \mathrm{C}$

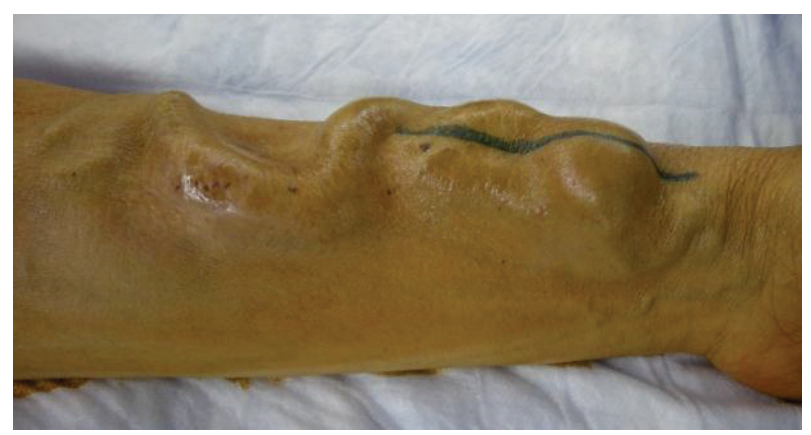

(a)

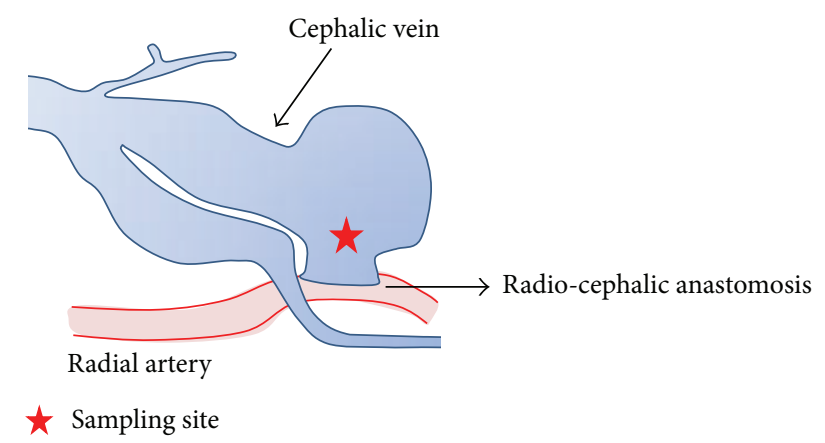

(b)

FIgUre 1: (a) Photograph of overflow AV fistula. (b) Schematic of overflow AV fistula.

with 5X First-Strand Buffer, 0.1 M DTT, 10 mM dNTP mix, and Affinity Script RNase Block Mix. The Affinity Script enzyme was inactivated at $70^{\circ} \mathrm{C}$ for $15 \mathrm{~min}$. The resulting cDNA products were then used as templates for in vitro transcription to generate fluorescent cRNA. They were mixed with a transcription master mix in the presence of T7 RNA polymerase and Cy3-labeled CTP and incubated at $40^{\circ} \mathrm{C}$ for 2 hours. Labeled cRNAs were purified using Qiagen's RNeasy Mini spin columns and eluted in $30 \mu \mathrm{L}$ of nuclease-free water. After amplification and labeling, cRNA quantity and cyanine incorporation were determined using a Nanodrop ND-1000 spectrophotometer and an Agilent Bioanalyzer.

\section{Sample Hybridization}

For each hybridization, $1.65 \mu \mathrm{g}$ of Cy3-labeled cRNA was fragmented and hybridized onto an Agilent Human GE $4 \mathrm{x} 44 \mathrm{~K}$ v2 Microarray (Design ID: 026652) for 17 hours at $65^{\circ} \mathrm{C}$. After washing, the microarrays were scanned using an Agilent DNA microarray scanner.

\section{Microarray Data Analysis}

The intensity values of each scanned feature were quantified using Agilent feature extraction software (version 10.7.3.1), which performs background subtractions. We only used features flagged as having no errors (present flags) and excluded features that were not positive, not significant, not 
TABLE 1: Patient characteristics.

\begin{tabular}{lccccc}
\hline Over flow AVF & Age & Gender & Cause of CRF & Patency period of AV fistula (months) & Blood flow (mL/min) \\
\hline 1 & 48 & M & CGN & 56 & 3790 \\
2 & 83 & F & CGN & 93 & 2760 \\
3 & 57 & M & CGN & 19 & 3280 \\
4 & 46 & M & CGN & 22 & 2710 \\
5 & 75 & M & CGN & 104 & 3520 \\
6 & 57 & F & IgA & 88 & 2340 \\
\hline Control & & & & & $(-)$ \\
1 & 67 & M & CGN & $(-)$ & $(-)$ \\
2 & 68 & F & CGN & $(-)$ & $(-)$ \\
3 & 56 & M & CGN & $(-)$ & $(-)$ \\
4 & 80 & F & CGN & $(-)$ & \\
\hline
\end{tabular}

TABLE 2: Genes significantly upregulated in the remodeled vein compared to the control vein (top 30).

\begin{tabular}{lccc}
\hline Probe name & P value & FCAbsolute & Gene symbol \\
\hline A_23_P106194 & 0.045653246 & 38.85 & FOS \\
A_23_P429998 & 0.021514166 & 27.22 & FOSB \\
A_24_P33895 & 0.001204797 & 25.26 & ATF3 \\
A_23_P46936 & $1.36 E-05$ & 24.77 & EGR2 \\
A_23_P96158 & 0.002943707 & 24.55 & KRT17 \\
A_23_P34915 & 0.001120758 & 23.39 & ATF3 \\
A_23_P71037 & 0.004629572 & 21.98 & IL6 \\
A_23_P46429 & $7.94 E-04$ & 21.04 & CYR61 \\
A_24_P882732 & 0.036205057 & 19.08 & \\
A_23_P97141 & 0.0211732 & 17.66 & RGS1 \\
A_23_P323751 & $9.21 E-05$ & 17.26 & FAM83D \\
A_33_P3316273 & 0.00369598 & 15.81 & CCL3 \\
A_23_P216225 & 0.0219925 & 15.72 & EGR3 \\
A_33_P3295203 & 0.001008955 & 15.65 & HAS1 \\
A_23_P131208 & $2.92 E-04$ & 14.26 & NR4A2 \\
A_23_P214080 & 0.00224604 & 13.9 & EGR1 \\
A_33_P3214105 & $8.77 E-04$ & 13.44 & ATF3 \\
A_33_P3390793 & 0.004063138 & 13.41 & TRIM36 \\
A_33_P3354607 & 0.001234311 & 13.09 & CCL4 \\
A_23_P79518 & 0.006241249 & 12.95 & IL1B \\
A_23_P1331 & 0.001146301 & 11.08 & COL13A1 \\
A_23_P110569 & $7.10 E-04$ & 10.2 & TRIM36 \\
A_23_P166408 & 0.003698398 & 10.04 & OSM \\
A_32_P76627 & $1.51 E-04$ & 10.02 & \\
A_23_P207564 & 0.00151482 & 9.94 & CCL4 \\
A_33_P3299066 & 0.001036557 & 9.61 & NR4A2 \\
A_33_P3214393 & 0.008276591 & 9.56 & \\
A_33_P3413741 & 0.032112285 & 9.53 & OXTR \\
A_33_P3271594 & 0.001451045 & 9.49 & TRIM54 \\
A_24_P158089 & 0.003357552 & 8.93 & \\
\hline & & & \\
\hline
\end{tabular}

uniform, not above background levels, saturated, or population outliers (marginal and absent flags). Normalization was performed using Agilent GeneSpring GX version 11.0.2. (per
TABLE 3: Genes significantly downregulated in the remodeled vein compared to the control vein (top 30).

\begin{tabular}{lccc}
\hline Probe name & FCAbsolute & $P$ value & Gene symbol \\
\hline A_23_P23783 & 18.99 & 0.009408315 & MYOC \\
A_23_P121545 & 14.63 & $6.67 E-04$ & GPM6A \\
A_33_P3368193 & 10.96 & $2.33 E-05$ & PNLIPRP3 \\
A_32_P92489 & 8.79 & 0.004908097 & PKD1L2 \\
A_24_P40626 & 8.15 & 0.011296479 & GREM2 \\
A_33_P3221408 & 8.14 & 0.004285622 & NTNG1 \\
A_23_P143526 & 7.15 & 0.004383178 & S100B \\
A_23_P136777 & 7.14 & $3.89 E-04$ & APOD \\
A_23_P102331 & 7.10 & 0.003490915 & SCN7A \\
A_33_P3421923 & 7.03 & 0.001119926 & CADM3 \\
A_23_P140384 & 7.00 & 0.026459113 & CTSG \\
A_33_P3363799 & 6.94 & 0.002682039 & NCAM1 \\
A_24_P203134 & 6.80 & 0.024163503 & DCAF12L1 \\
A_24_P280684 & 6.69 & 0.03005707 & FBXO40 \\
A_23_P55544 & 6.51 & 0.004269639 & CCBE1 \\
A_23_P73571 & 6.45 & 0.03981546 & MUM1L1 \\
A_23_P212050 & 6.22 & 0.021448081 & BCHE \\
A_33_P3336557 & 6.12 & $1.20 E-04$ & \\
A_23_P121676 & 6.07 & 0.014616995 & CXXC4 \\
A_23_P204885 & 6.01 & 0.007333652 & PCDH20 \\
A_23_P64919 & 5.92 & 0.012492463 & RERGL \\
A_23_P422911 & 5.81 & $6.12 E-04$ & HS6ST3 \\
A_23_P146233 & 5.79 & 0.01808302 & LPL \\
A_23_P110624 & 5.76 & 0.003615111 & CTNND2 \\
A_23_P45185 & 5.69 & 0.00549277 & FIGF \\
A_23_P110764 & 5.65 & 0.009343005 & MYOT \\
A_23_P114862 & 5.41 & 0.039056532 & ANGPTL7 \\
A_23_P39251 & 5.31 & $9.92 E-04$ & PLIN5 \\
A_23_P111402 & 5.28 & 0.008291814 & RSPO3 \\
A_33_P3400763 & 5.26 & 0.038730744 & PLIN4 \\
\hline
\end{tabular}

chip: normalization to the 75 percentile shift; per gene: normalization to median across all samples). There are 34,127 probes in total on the Agilent Human GE $4 \times 44 \mathrm{~K}$ v2 
TABLE 4: Statistically overrepresented GO terms in the biological process category $(P<0.001)$.

\begin{tabular}{|c|c|c|c|c|c|c|}
\hline \multicolumn{7}{|c|}{ Biological process } \\
\hline $\begin{array}{l}\text { GO accession } \\
\text { (with AmiGO link) }\end{array}$ & GO term & Corrected $P$ value & $\begin{array}{l}\text { Count in } \\
\text { selection }\end{array}$ & $\begin{array}{l}\% \text { count in } \\
\text { selection }\end{array}$ & $\begin{array}{l}\text { Count } \\
\text { in total }\end{array}$ & $\begin{array}{l}\% \text { count } \\
\text { in total }\end{array}$ \\
\hline GO:0032502 & Developmental process & $2.100 E-11$ & 77 & 29.8 & 3077 & 17.9 \\
\hline GO:0007275 & Multicellular organismal development & $1.340 E-10$ & 67 & 26.0 & 2810 & 16.3 \\
\hline GO:0010033 & Response to organic substance & $3.530 E-10$ & 40 & 15.5 & 698 & 4.1 \\
\hline GO:0001568 & Blood vessel development & $9.230 E-10$ & 21 & 8.1 & 231 & 1.3 \\
\hline GO:0048514 & Blood vessel morphogenesis & $1.250 E-09$ & 19 & 7.4 & 198 & 1.1 \\
\hline GO:0001944 & Vasculature development & $1.250 E-09$ & 21 & 8.1 & 238 & 1.4 \\
\hline GO:0048545 & Response to steroid hormone stimulus & $1.360 E-09$ & 23 & 8.9 & 183 & 1.1 \\
\hline GO:0001525 & Angiogenesis & $2.930 E-09$ & 18 & 7.0 & 139 & 0.8 \\
\hline GO:0009653 & Anatomical structure morphogenesis & $4.930 E-09$ & 30 & 11.6 & 1125 & 6.5 \\
\hline GO:0016265 & Death & $5.110 E-09$ & 39 & 15.1 & 663 & 3.8 \\
\hline GO:0048646 & $\begin{array}{l}\text { Anatomical structure formation } \\
\text { involved in morphogenesis }\end{array}$ & $5.950 E-09$ & 18 & 7.0 & 306 & 1.8 \\
\hline GO:0008219 & Cell death & $1.280 E-08$ & 37 & 14.3 & 658 & 3.8 \\
\hline GO:0042221 & Response to chemical stimulus & $1.280 E-08$ & 53 & 20.5 & 1264 & 7.3 \\
\hline GO:0048856 & Anatomical structure development & $1.320 E-08$ & 42 & 16.3 & 2437 & 14.1 \\
\hline GO:0006950 & Response to stress & $2.080 E-08$ & 51 & 19.8 & 1642 & 9.5 \\
\hline GO:0048731 & System development & $3.600 E-08$ & 38 & 14.7 & 2284 & 13.3 \\
\hline GO:0032570 & Response to progesterone stimulus & $7.370 E-08$ & 9 & 3.5 & 21 & 0.1 \\
\hline GO:0006915|GO:0008632 & Apoptosis & $1.340 E-07$ & 31 & 12.0 & 541 & 3.1 \\
\hline GO:0012501|GO:0016244 & Programmed cell death & $1.900 E-07$ & 31 & 12.0 & 549 & 3.2 \\
\hline GO:0042981 & Regulation of apoptosis & $2.470 E-07$ & 35 & 13.6 & 796 & 4.6 \\
\hline GO:0032501|GO:0050874 & Multicellular organismal process & $3.190 E-07$ & 70 & 27.1 & 4154 & 24.1 \\
\hline GO:0043067|GO:0043070 & Regulation of programmed cell death & $3.190 E-07$ & 35 & 13.6 & 804 & 4.7 \\
\hline GO:0010941 & Regulation of cell death & $3.310 E-07$ & 35 & 13.6 & 807 & 4.7 \\
\hline GO:0009887 & Organ morphogenesis & $3.510 E-07$ & 26 & 10.1 & 685 & 4.0 \\
\hline GO:0009628 & Response to abiotic stimulus & $5.270 E-07$ & 16 & 6.2 & 357 & 2.1 \\
\hline GO:0009725 & Response to hormone stimulus & $5.390 E-07$ & 23 & 8.9 & 358 & 2.1 \\
\hline GO:0048519|GO:0043118 & Negative regulation of biological process & $6.290 E-07$ & 39 & 15.1 & 1756 & 10.2 \\
\hline GO:0009719 & Response to endogenous stimulus & $7.450 E-07$ & 24 & 9.3 & 391 & 2.3 \\
\hline GO:0009605 & Response to external stimulus & $7.730 E-07$ & 32 & 12.4 & 869 & 5.0 \\
\hline GO:0048513 & Organ development & $1.510 E-06$ & 34 & 13.2 & 1682 & 9.8 \\
\hline GO:0009607 & Response to biotic stimulus & $2.160 E-06$ & 23 & 8.9 & 385 & 2.2 \\
\hline GO:0070482 & Response to oxygen levels & $3.460 E-06$ & 14 & 5.4 & 137 & 0.8 \\
\hline GO:0009266 & Response to temperature stimulus & $3.950 E-06$ & 9 & 3.5 & 86 & 0.5 \\
\hline GO:0050896|GO:0051869 & Response to stimulus & $6.040 E-06$ & 89 & 34.5 & 3356 & 19.5 \\
\hline GO:0048523|GO:0051243 & Negative regulation of cellular process & $8.220 E-06$ & 38 & 14.7 & 1606 & 9.3 \\
\hline GO:0009408|GO:0006951 & Response to heat & $8.220 E-06$ & 9 & 3.5 & 61 & 0.4 \\
\hline GO:0006928 & Cellular component movement & $1.200 E-05$ & 14 & 5.4 & 450 & 2.6 \\
\hline GO:0050793 & Regulation of developmental process & $1.590 E-05$ & 8 & 3.1 & 670 & 3.9 \\
\hline GO:0048869 & Cellular developmental process & $1.600 E-05$ & 24 & 9.3 & 1641 & 9.5 \\
\hline GO:0022603 & $\begin{array}{l}\text { Regulation of anatomical structure } \\
\text { morphogenesis }\end{array}$ & $2.530 E-05$ & 5 & 1.9 & 228 & 1.3 \\
\hline GO:0051239 & $\begin{array}{l}\text { Regulation of multicellular organismal } \\
\text { process }\end{array}$ & $2.860 E-05$ & 7 & 2.7 & 924 & 5.4 \\
\hline GO:0007565 & Female pregnancy & $3.100 E-05$ & 13 & 5.0 & 104 & 0.6 \\
\hline GO:0030154 & Cell differentiation & $4.450 E-05$ & 24 & 9.3 & 1576 & 9.1 \\
\hline GO:0042127 & Regulation of cell proliferation & $4.830 E-05$ & 29 & 11.2 & 773 & 4.5 \\
\hline
\end{tabular}


TABLE 4: Continued.

\begin{tabular}{|c|c|c|c|c|c|c|}
\hline \multicolumn{7}{|c|}{ Biological process } \\
\hline $\begin{array}{l}\text { GO accession } \\
\text { (with AmiGO link) }\end{array}$ & GO term & $\begin{array}{l}\text { Corrected } \\
P \text { value }\end{array}$ & $\begin{array}{l}\text { Count in } \\
\text { selection }\end{array}$ & $\begin{array}{l}\% \text { count in } \\
\text { selection }\end{array}$ & $\begin{array}{l}\text { Count in } \\
\text { total }\end{array}$ & $\begin{array}{l}\% \text { count } \\
\text { in total }\end{array}$ \\
\hline GO:0001666 & Response to hypoxia & $6.420 E-05$ & 14 & 5.4 & 131 & 0.8 \\
\hline GO:0008284 & $\begin{array}{l}\text { Positive regulation of cell } \\
\text { proliferation }\end{array}$ & $7.720 E-05$ & 16 & 6.2 & 410 & 2.4 \\
\hline GO:0048522|GO:0051242 & Positive regulation of cellular process & $7.720 E-05$ & 34 & 13.2 & 1806 & 10.5 \\
\hline GO:0051789 & Response to protein stimulus & $8.360 E-05$ & 11 & 4.3 & 96 & 0.6 \\
\hline GO:0048518|GO:0043119 & $\begin{array}{l}\text { Positive regulation of biological } \\
\text { process }\end{array}$ & $9.260 E-05$ & 35 & 13.6 & 1982 & 11.5 \\
\hline GO:0043627 & Response to estrogen stimulus & $1.000 E-04$ & 12 & 4.7 & 98 & 0.6 \\
\hline GO:0009991 & Response to extracellular stimulus & $1.000 E-04$ & 6 & 2.3 & 204 & 1.2 \\
\hline GO:0042493|GO:0017035 & Response to drug & $1.770 E-04$ & 17 & 6.6 & 213 & 1.2 \\
\hline GO:0043066 & Negative regulation of apoptosis & $1.790 E-04$ & 19 & 7.4 & 345 & 2.0 \\
\hline GO:0043069|GO:0043072 & $\begin{array}{l}\text { Negative regulation of programmed } \\
\text { cell death }\end{array}$ & $2.200 E-04$ & 19 & 7.4 & 350 & 2.0 \\
\hline GO:0051384 & Response to glucocorticoid stimulus & $2.200 E-04$ & 10 & 3.9 & 70 & 0.4 \\
\hline GO:0050789|GO:0050791 & Regulation of biological process & $2.440 E-04$ & 109 & 42.2 & 7200 & 41.8 \\
\hline GO:0060548 & Negative regulation of cell death & $2.570 E-04$ & 19 & 7.4 & 354 & 2.1 \\
\hline GO:0051707|GO:0009613|GO:0042828 & Response to other organism & $2.770 E-04$ & 17 & 6.6 & 300 & 1.7 \\
\hline GO:0040011 & Locomotion & $2.800 E-04$ & 16 & 6.2 & 415 & 2.4 \\
\hline GO:0009611|GO:0002245 & Response to wounding & $2.800 E-04$ & 21 & 8.1 & 507 & 2.9 \\
\hline GO:0031960 & Response to corticosteroid stimulus & $3.860 E-04$ & 10 & 3.9 & 75 & 0.4 \\
\hline GO:0050794|GO:0051244 & Regulation of cellular process & $4.070 E-04$ & 108 & 41.9 & 6938 & 40.3 \\
\hline GO:0014070 & Response to organic cyclic substance & $4.200 E-04$ & 12 & 4.7 & 114 & 0.7 \\
\hline GO:0051128 & $\begin{array}{l}\text { Regulation of cellular component } \\
\text { organization }\end{array}$ & $5.900 E-04$ & 6 & 2.3 & 466 & 2.7 \\
\hline GO:0065007 & Biological regulation & $7.620 E-04$ & 109 & 42.2 & 7592 & 44.1 \\
\hline GO:0051704|GO:0051706 & Multiorganism process & $7.900 E-04$ & 27 & 10.5 & 706 & 4.1 \\
\hline GO:0031099 & Regeneration & $8.770 E-04$ & 6 & 2.3 & 65 & 0.4 \\
\hline GO:0007610 & Behavior & $9.680 E-04$ & 12 & 4.7 & 449 & 2.6 \\
\hline
\end{tabular}

TABLE 5: Statistically overrepresented GO terms in the molecular function category $(P<0.01)$.

\begin{tabular}{|c|c|c|c|c|c|c|}
\hline \multicolumn{7}{|c|}{ Molecular function } \\
\hline $\begin{array}{l}\text { GO accession } \\
\text { (with AmiGO link) }\end{array}$ & GO term & Corrected $P$ value & $\begin{array}{l}\text { Count in } \\
\text { selection }\end{array}$ & $\begin{array}{l}\% \text { count in } \\
\text { selection }\end{array}$ & $\begin{array}{c}\text { Count in } \\
\text { total }\end{array}$ & $\begin{array}{l}\% \text { count in } \\
\text { total }\end{array}$ \\
\hline GO:0005539 & Glycosaminoglycan binding & $9.960 E-06$ & 14 & 5.4 & 149 & 0.9 \\
\hline GO:0005515|GO:0045308 & Protein binding & $1.550 E-05$ & 170 & 65.9 & 8104 & 47.0 \\
\hline GO:0001871 & Pattern binding & $3.120 E-05$ & 14 & 5.4 & 164 & 1.0 \\
\hline GO:0030247 & Polysaccharide binding & $3.120 E-05$ & 14 & 5.4 & 164 & 1.0 \\
\hline GO:0008201 & Heparin binding & $6.710 E-05$ & 13 & 5.0 & 112 & 0.7 \\
\hline GO:0005126 & Cytokine receptor binding & $7.930 E-05$ & 4 & 1.6 & 177 & 1.0 \\
\hline GO:0005125 & Cytokine activity & $2.200 E-04$ & 12 & 4.7 & 193 & 1.1 \\
\hline GO:0005114 & $\begin{array}{l}\text { Type II transforming growth } \\
\text { factor beta receptor binding }\end{array}$ & $1.143 E-03$ & 4 & 1.6 & 7 & 0.0 \\
\hline GO:0008083 & Growth factor activity & $2.365 E-03$ & 13 & 5.0 & 160 & 0.9 \\
\hline GO:0005102 & Receptor binding & $3.106 E-03$ & 24 & 9.3 & 873 & 5.1 \\
\hline GO:0030246 & Carbohydrate binding & $7.049 E-03$ & 14 & 5.4 & 354 & 2.1 \\
\hline
\end{tabular}


TABLE 6: Statistically overrepresented GO terms in the cellular component category $(P<0.01)$.

\begin{tabular}{|c|c|c|c|c|c|c|}
\hline \multicolumn{7}{|c|}{ Cellular component } \\
\hline $\begin{array}{l}\text { GO accession } \\
\text { (with AmiGO link) }\end{array}$ & GO term & $\begin{array}{l}\text { Corrected } \\
P \text { value }\end{array}$ & $\begin{array}{l}\text { Count in } \\
\text { selection }\end{array}$ & $\begin{array}{l}\% \text { count in } \\
\text { selection }\end{array}$ & Count in total & $\begin{array}{l}\% \text { count in } \\
\text { total }\end{array}$ \\
\hline GO:0044421 & Extracellular region part & $2.190 E-09$ & 49 & 19.0 & 937 & 5.4 \\
\hline GO:0031012 & Extracellular matrix & $7.420 E-07$ & 25 & 9.7 & 339 & 2.0 \\
\hline GO:0005576 & Extracellular region & $3.880 E-06$ & 69 & 26.7 & 1923 & 11.2 \\
\hline GO:0005615 & Extracellular space & $1.690 E-05$ & 32 & 12.4 & 673 & 3.9 \\
\hline GO:0005578 & Proteinaceous extracellular matrix & $1.200 E-04$ & 20 & 7.8 & 309 & 1.8 \\
\hline GO:0060205 & $\begin{array}{l}\text { Cytoplasmic membrane-bounded } \\
\text { vesicle lumen }\end{array}$ & $4.070 E-04$ & 7 & 2.7 & 44 & 0.3 \\
\hline GO:0031983 & Vesicle lumen & $5.660 E-04$ & 7 & 2.7 & 46 & 0.3 \\
\hline GO:0031093 & Platelet alpha granule lumen & $2.468 E-03$ & 7 & 2.7 & 41 & 0.2 \\
\hline
\end{tabular}

Microarray (Design ID: 026652), excluding control probes. The microarray data were submitted to NCBI GEO (http:// www.ncbi.nlm.nih.gov/geo/), sample number [GSE39488].

The altered transcripts were quantified using a comparative method. We applied a $P$ value $<0.05$ combined with a $>2$-fold change in normalized intensity to identify genes with significantly different expression patterns.

\section{Gene Ontology Analysis and Pathway Analysis}

The gene ontology analysis was performed using Agilent Technologies GeneSpring GX software (11.0.2). Pathway analysis was performed with GenMAPP 2.1 (http://www.genmapp.org/).

\section{Results}

The cDNA microarray analysis revealed that 397 genes were upregulated and 456 were downregulated (Tables 2 and 3 ).

The gene ontology analysis revealed that biological developmental processes and glycosaminoglycan binding were the most upregulated. In addition, most upregulation occurred extracellularly (Tables 4, 5, and 6).

The pathway analysis revealed that the TGF beta signaling pathway, cytokines and inflammatory response pathway, hypertrophy model, and the myometrial relaxation and contraction pathway were upregulated (Table 7).

\section{Discussion}

AV fistulas are very useful for determining the optimal blood flow for hemodialysis since satisfactory blood access flow is necessary for adequate hemodialysis. When stenotic lesions occur within the vascular system and blood flow is insufficient, a percutaneous transluminal angioplasty or some other intervention is performed. However, overflow AV fistulas increase cardiac output and cause high-output cardiac failure [1].
In the 2005 Japanese Society for Dialysis Therapy Guidelines for Vascular Access Construction and Repair for Chronic Hemodialysis, vascular access flow is said to lead to heart failure when the blood access flow is greater than 1.0-1.5 L/ min or when the vascular access flow/cardiac output ratio is $>20 \%$ [1]. If the vascular access flow is clearly responsible for a decline in cardiac function, then it is necessary to intentionally constrict or occlude the vascular access [1]. Surveillance of blood flow in internal shunts by the Doppler echocardiography has become widespread and overflow AV fistulas are now actively treated. Several recent studies have noted the importance of histological changes in AV fistulas $[4,5]$.

Microarrays of vascular access have been reported in experimental animal models, but there have been no such analyses in humans [6]. In the present study, venous tissue samples were resected from overflow AV fistulas from 6 hemodialysis patients and from the normal cephalic veins of 4 other hemodialysis patients, and their gene expression patterns were compared.

It is interesting to note that zinc finger-containing transcription factors such as egrl, egr2, and egr3 and immediate early genes such as fos and jun, were found to be remarkably upregulated in the present study; egrl, egr 2, and egr 3 have been implicated in the proliferation and differentiation of many cell types $[7,8]$, and fos and jun have been linked to the regulation of angiogenesis [9]. Moreover, egr-1, c-jun, and cfos have been linked to the regulation of free radical scavenging enzymes [10-13]. We also observed the upregulation of free radical scavenging enzyme activity in the walls of the overflow AV fistulas, which may reflect chronic reactive oxygen species formation in overflow AV fistulas.

The pathway analysis indicated that the TGF beta signaling pathway and cytokines and inflammatory response pathway were upregulated. This suggests that overflow AV fistulas may be implicated in chronic inflammation in hemodialysis patients.

Malnutrition, inflammation, and atherosclerosis (MIA syndrome) are common in end-stage renal disease (ESRD) patients, and inflammation has been identified as playing a key role in atherosclerotic cardiovascular disease. Proinflammatory cytokines are pivotal to the inflammation that is, 
TABLE 7: Pathway analysis results.

\begin{tabular}{|c|c|}
\hline Pathway name & LS_vs_control \\
\hline Alpha6 beta 4 integrin signaling pathway & 0.793 \\
\hline Androgen receptor signaling pathway & 0.528 \\
\hline Apoptosis mechanisms & 0.124 \\
\hline B-cell receptor signaling pathway & 0.023 \\
\hline G1 to $S$ cell cycle control & 1 \\
\hline Cell cycle & 0.487 \\
\hline Delta-Notch signaling pathway & 0.226 \\
\hline DNA replication & 1 \\
\hline EGFR1 signaling pathway & 0.856 \\
\hline $\begin{array}{l}\text { FAS pathway and stress induction of HSP } \\
\text { regulation }\end{array}$ & 1 \\
\hline Focal Adhesion & 0.003 \\
\hline G13 signaling pathway & 0.269 \\
\hline G protein signaling pathways & 0.258 \\
\hline Hedgehog signaling pathway & 1 \\
\hline Apoptosis modulation by HSP70 & 1 \\
\hline Id signaling pathway & 1 \\
\hline IL-1 signaling pathway & 1 \\
\hline IL-2 signaling pathway & 0.327 \\
\hline IL-3 signaling pathway & 0.371 \\
\hline IL-4 signaling pathway & 0.589 \\
\hline IL-5 signaling pathway & 0.576 \\
\hline IL-6 signaling pathway & 1 \\
\hline IL-7 signaling pathway & 0.498 \\
\hline IL-9 signaling pathway & 1 \\
\hline Human insulin signaling & 0.387 \\
\hline Integrin-mediated cell adhesion & 0.363 \\
\hline Kit receptor signaling pathway & 0.051 \\
\hline MAPK cascade & 1 \\
\hline MAPK signaling pathway & 0.011 \\
\hline mRNA processing (Homo sapiens) & 0.014 \\
\hline Notch signaling pathway & 0.191 \\
\hline Ovarian infertility genes & 1 \\
\hline p38 MAPK signaling pathway (BioCarta) & 0.108 \\
\hline Regulation of actin cytoskeleton & 0.834 \\
\hline Eukaryotic transcription initiation & 0.511 \\
\hline Signal transduction of S1P & 0.384 \\
\hline $\begin{array}{l}\text { Signaling of hepatocyte growth factor } \\
\text { receptor }\end{array}$ & 1 \\
\hline $\mathrm{T}$ cell receptor signaling pathway & 0.243 \\
\hline TGF-beta receptor signaling pathway & 0.095 \\
\hline TGF beta signaling pathway & 0 \\
\hline TNF-alpha/NF- $\kappa$ B signaling pathway & 0.752 \\
\hline Translation factors & 0.368 \\
\hline Wnt signaling pathway & 0.15 \\
\hline Wnt signaling pathway & 0.051 \\
\hline Acetylcholine synthesis & 1 \\
\hline Alanine and aspartate metabolism & - \\
\hline Biogenic amine synthesis & 1 \\
\hline
\end{tabular}

TABLE 7: Continued.

\begin{tabular}{|c|c|}
\hline Pathway name & LS_vs_control \\
\hline Cholesterol biosynthesis & 0.644 \\
\hline Eicosanoid synthesis & 1 \\
\hline Electron transport chain & 0.013 \\
\hline Fatty acid beta oxidation 1 & 0.403 \\
\hline Fatty acid beta oxidation 2 & 1 \\
\hline Fatty acid beta oxidation 3 & 1 \\
\hline Beta oxidation meta MAPP & 0.264 \\
\hline Fatty acid omega oxidation & 0.687 \\
\hline Fatty acid biosynthesis & 0.426 \\
\hline $\begin{array}{l}\text { Glucocorticoid and mineralcorticoid } \\
\text { metabolism }\end{array}$ & 1 \\
\hline Glutathione metabolism & 0.399 \\
\hline Glycogen metabolism & 0.261 \\
\hline Glycolysis and gluconeogenesis & 0.235 \\
\hline Heme biosynthesis & 1 \\
\hline TCA cycle & 0.24 \\
\hline Mitochondrial LC-fatty acid beta-oxidation & 0.635 \\
\hline $\begin{array}{l}\text { Nuclear receptors in lipid metabolism and } \\
\text { toxicity }\end{array}$ & 0.389 \\
\hline Nucleotide metabolism & 0.622 \\
\hline Pentose phosphate pathway & 1 \\
\hline Prostaglandin synthesis and regulation & 1 \\
\hline Statin pathway (PharmGKB) & 1 \\
\hline Steroid biosynthesis & 1 \\
\hline Synthesis and degradation of ketone bodies & 1 \\
\hline Triacylglyceride synthesis & 0.419 \\
\hline Tryptophan metabolism & 0.501 \\
\hline Beta oxidation of unsaturated fatty acids & 1 \\
\hline Urea cycle and metabolism of amino groups & - \\
\hline GPCRs, class A rhodopsin-like & 0.317 \\
\hline GPCRs, class B secretin-like & 1 \\
\hline $\begin{array}{l}\text { GPCRs, class } \mathrm{C} \text { metabotropic glutamate, } \\
\text { pheromone }\end{array}$ & 1 \\
\hline GPCRs, other & 1 \\
\hline Matrix metalloproteinases & 0.652 \\
\hline Monoamine GPCRs & 1 \\
\hline Nuclear receptors & 1 \\
\hline Nucleotide GPCRs & 1 \\
\hline Peptide GPCRs & 0.081 \\
\hline Cytoplasmic ribosomal proteins & 0.025 \\
\hline Small ligand GPCRs & 1 \\
\hline ACE inhibitor pathway (Homo sapiens) & 0.254 \\
\hline Adipogenesis human & 0.101 \\
\hline Blood clotting cascade & 0.155 \\
\hline Calcium regulation in the cardiac cell & 0.431 \\
\hline Circadian exercise & 0.754 \\
\hline $\begin{array}{l}\text { Complement activation and classical } \\
\text { pathway }\end{array}$ & 0.646 \\
\hline $\begin{array}{l}\text { Complement activation and classical } \\
\text { pathway }\end{array}$ & 0.022 \\
\hline
\end{tabular}


TABle 7: Continued.

\begin{tabular}{lc}
\hline Pathway name & LS_vs_control \\
\hline $\begin{array}{l}\text { Cytokines and inflammatory response } \\
\text { (BioCarta) }\end{array}$ & $\mathbf{0}$ \\
Hypertrophy model & $\mathbf{0}$ \\
Inflammatory response pathway & 0.054 \\
Irinotecan pathway (Homo sapiens) & 0.685 \\
Oxidative stress & 0.402 \\
Proteasome degradation & 0.278 \\
Myometrial relaxation and contraction & $\mathbf{0}$ \\
pathways & 0.427 \\
\hline
\end{tabular}

associated with malnutrition and atherosclerosis in ESRD [14]. Our findings suggest that overflow AV fistulas may be implicated in MIA syndrome.

\section{Conclusion}

Combining microarray results and pathway information available via the Internet provided biological insight into molecular changes in the venous walls of overflow AV fistulas. Despite the small sample size, our study findings suggest that overflow AV fistulas may be implicated in chronic inflammation in hemodialysis patients.

\section{References}

[1] S. Ohira, H. Naito, I. Amano et al., "2005 Japanese Society for Dialysis Therapy guidelines for vascular access construction and repair for chronic hemodialysis," Therapeutic Apheresis and Dialysis, vol. 10, no. 5, pp. 449-462, 2006.

[2] C. Basile, C. Lomonte, L. Vernaglione, F. Casucci, M. Antonelli, and N. Losurdo, "The relationship between the flow of arteriovenous fistula and cardiac output in haemodialysis patients," Nephrology Dialysis Transplantation, vol. 23, no. 1, pp. 282-287, 2008.

[3] N. M. Krivitski, "Theory and validation of access flow measurement by dilution technique during hemodialysis," Kidney International, vol. 48, no. 1, pp. 244-250, 1995.

[4] T. Lee and P. Roy-Chaudhury, "Advances and new frontiers in the pathophysiology of venous neointimal hyperplasia and dialysis access stenosis," Advances in Chronic Kidney Disease, vol. 16, no. 5, pp. 329-338, 2009.

[5] P. Roy-Chaudhury, Y. Wang, M. Krishnamoorthy et al., "Cellular phenotypes in human stenotic lesions from haemodialysis vascular access," Nephrology Dialysis Transplantation, vol. 24, no. 9, pp. 2786-2791, 2009.

[6] D. Abeles, S. Kwei, G. Stavrakis, Y. Zhang, E. T. Wang, and G. García-Cardeña, "Gene expression changes evoked in a venous segment exposed to arterial flow," Journal of Vascular Surgery, vol. 44, no. 4, pp. 863-870, 2006.

[7] K. B. Boyle, D. Hadaschik, S. Virtue et al., "The transcription factors Egr1 and Egr2 have opposing influences on adipocyte differentiation," Cell Death and Differentiation, vol. 16, no. 5, pp. 782-789, 2009.
[8] J. Kumbrink, K. H. Kirsch, and J. P. Johnson, "EGR1, EGR2, and EGR3 activate the expression of their coregulator NAB2 establishing a negative feedback loop in cells of neuroectodermal and epithelial origin," Journal of Cellular Biochemistry, vol. 111, no. 1, pp. 207-217, 2010.

[9] L. Marconcini, S. Marchio, L. Morbidelli et al., "c-fos-Induced growth factor/vascular endothelial growth factor D induces angiogenesis in vivo and in vitro," Proceedings of the National Academy of Sciences of the United States of America, vol. 96, no. 17, pp. 9671-9676, 1999.

[10] V. Schettler, K. Völker, E. G. Schulz, and E. Wieland, "Impact of lipid apheresis on Egr-1, c-Jun, c-Fos, and Hsp70 gene expression in white blood cells," Therapeutic Apheresis and Dialysis, vol. 15, no. 1, pp. 105-112, 2011.

[11] K. Maehara, K. Oh-Hashi, and K. I. Isobe, "Early growth-responsive-1-dependent manganese superoxide dismutase gene transcription mediated by platelet-derived growth factor," The FASEB Journal, vol. 15, no. 11, pp. 2025-2026, 2001.

[12] J. Wenk, P. Brenneisen, M. Wlaschek et al., "Stable overexpression of manganese superoxide dismutase in mitochondria identifies hydrogen peroxide as a major oxidant in the AP-1-mediated induction of matrix-degrading metalloprotease-1," The Journal of Biological Chemistry, vol. 274, no. 36, pp. 2586925876, 1999.

[13] T. Kondo, F. R. Sharp, J. Honkaniemi, S. Mikawa, C. J. Epstein, and P. H. Chan, "DNA fragmentation and prolonged expression of c-fos, c-jun, and hsp70 in kainic acid-induced neuronal cell death in transgenic mice overexpressing human CuZn-superoxide dismutase," Journal of Cerebral Blood Flow and Metabolism, vol. 17, no. 3, pp. 241-256, 1997.

[14] R. Pecoits-Filho, B. Lindholm, and P. Stenvinkel, "The malnutrition, inflammation, and atherosclerosis (MIA) syndrome-the heart of the matter," Nephrology Dialysis Transplantation, vol. 17, supplement 11, pp. 28-31, 2002. 


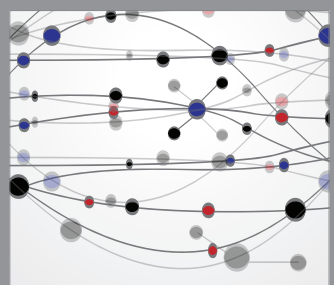

The Scientific World Journal
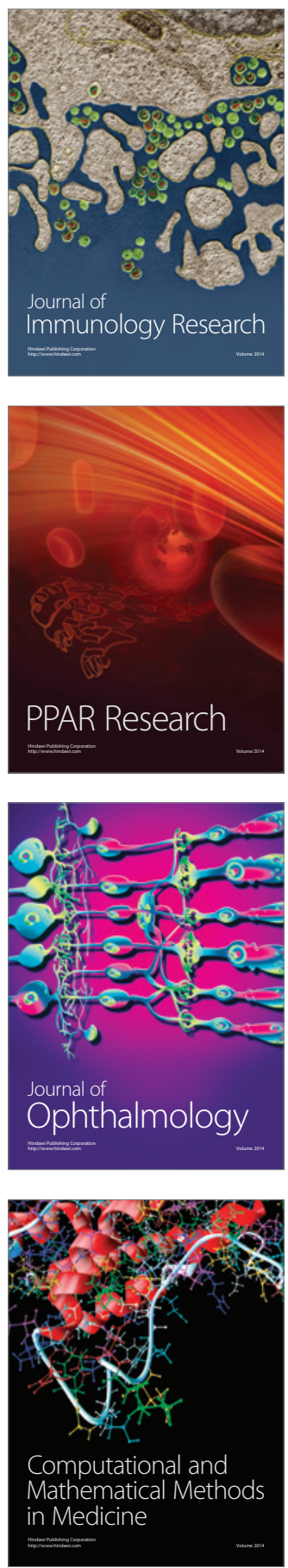

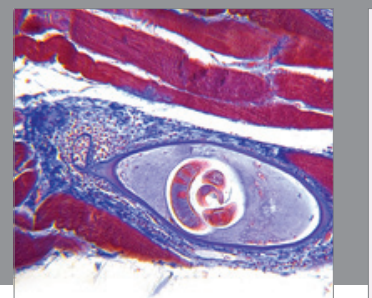

Gastroenterology

Research and Practice
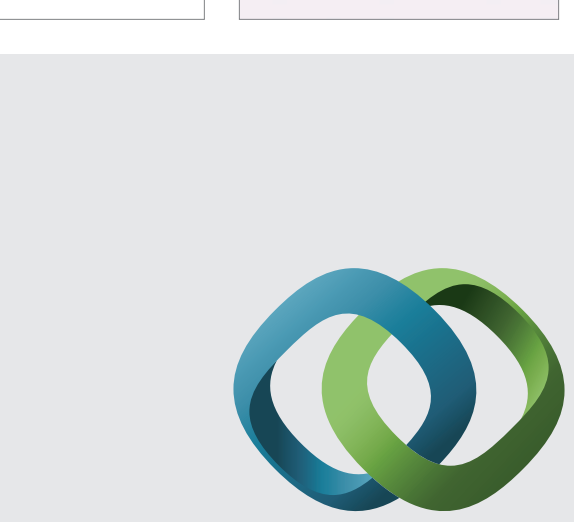

\section{Hindawi}

Submit your manuscripts at

http://www.hindawi.com
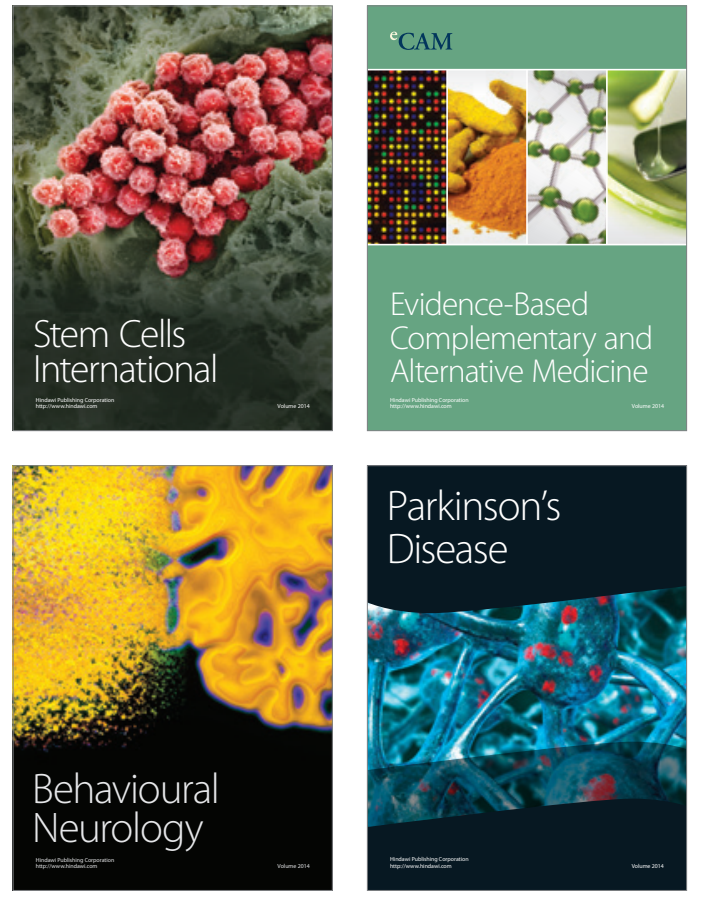
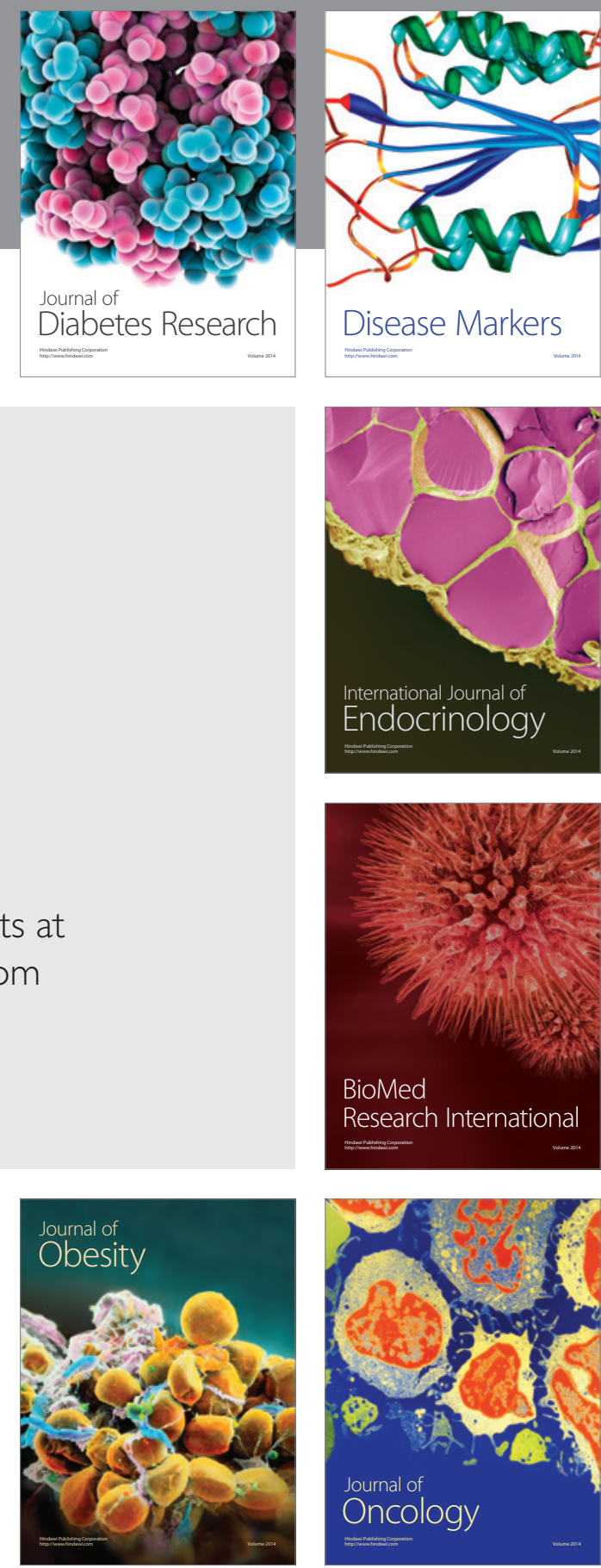

Disease Markers
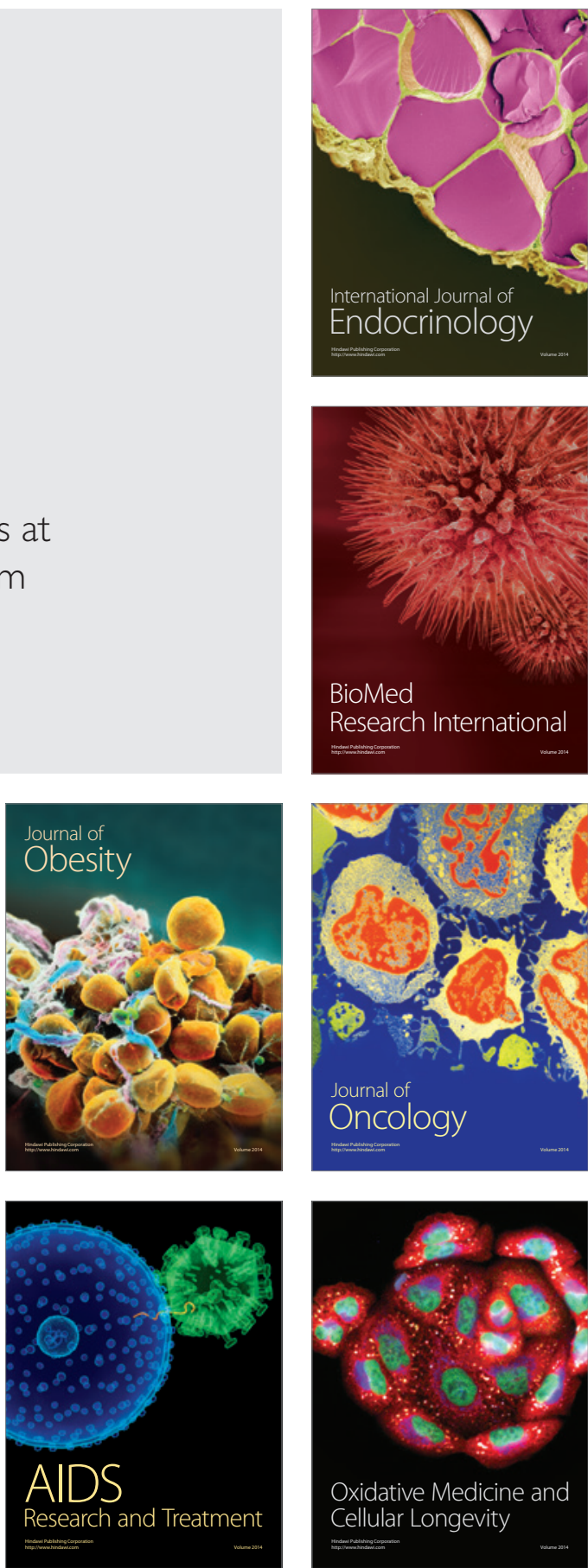